\title{
Editorial
}

Digestion

\section{Pathophysiology of Acute Pancreatitis}

\author{
If You Believe in Mice - It's Time for Conditional Gene Targeting!
}

\author{
Roland M. Schmid \\ II. Medizinische Klinik, Klinikum rechts der Isar, Technische Universität München, München, Deutschland
}

Acute pancreatitis is a common condition with a selflimiting course in most cases and a severe form associated with a significant risk of morbidity and mortality. High mortality is due to the systemic inflammatory response syndrome (SIRS) leading to multiple organ failure (MOF) [1]. The study of early events in acute pancreatitis in humans is restricted to the model of endoscopic retrograde cholangiopancreatography (ERCP)-induced pancreatitis and limited to imaging studies and measurement of biochemical markers in the serum including cytokines. The anatomic position and relationships of the pancreas make direct observation of pancreatic pathology and biopsies difficult or almost impossible. Therefore, most of our pathophysiological concepts are based on animals studies.

Several animal models of acute pancreatitis have been developed mostly in rodents, although these models fail to reproduce the human disease. Gallstone-induced acute pancreatitis cannot be reproduced in mice or rats. Acute or chronic ethanol feeding does not lead to acute pancreatitis. Nevertheless, there are experimental models which mimic some aspects of acute pancreatitis in humans. A mild self-limiting form of acute pancreatitis in rodents can be induced by injection of the secretagogue cerulein in supramaximal doses. This model has been utilized to study events inside the acinar cell including activation of digestive proenzymes, alterations of the cytoskeleton, activation of signaling pathways and synthesis of cytokines. In rats severe acute pancreatitis can be produced by injec- tion of bile acid into the main pancreatic duct. In CD-1 mice severe acute pancreatitis can be induced with a choline-deficient, ethionine-supplemented diet. These models are mostly used to investigate the systemic inflammatory response, multiorgan failure and to evaluate therapeutic interventions. Although all these models have in common that they do not recapitulate the human disease, we use them as tools and hope that they are helpful in understanding the pathophysiology of acute pancreatitis in humans. It is important to keep in mind that there is no proof that these models are predictive for the pathophysiology or treatment of the human disease.

The pathogenesis of acute pancreatitis is far from being understood. There is good evidence that autodigestion of the gland is involved, however, the initial trigger which converts digestive proenzymes into their active forms is unknown. As pancreatitis starts, a pancreatic defense program is initiated involving important reorientation of the gene expression pattern within the acinar cell. Furthermore, the relationship between pancreatic injury and the uncontrolled systemic inflammatory response is unclear. In the last few years a number of signaling pathways have been identified to be activated in the course of acute pancreatitis [2-4]. At least some of these signaling cascades are induced in a rapid fashion with a kinetic similar to the activation of proenzymes [5]. On the one hand, these pathways might contribute to the pancreatic defense program; on the other hand, they may trigger the synthesis of pro-inflammatory cytokines.

\section{KARGER \\ Fax +4161306 1234 E-Mail karger@karger.ch} www.karger.com (c) 2005 S. Karger AG, Basel 0012-2823/05/0713-0159\$22.00/0

Accessible online at: www.karger.com/dig
Roland M. Schmid, MD

II. Medizinische Klinik, Klinikum rechts der Isar, Technische Universität München Ismaninger Strasse 22

DE-81675 München (Germany) 
The development of techniques to genetically engineer mice has revolutionized biology and allows a new approach to study the pathophysiology of acute experimental pancreatitis. Genes can be inactivated in the germline by homologous recombination resulting in classical knockout mice. Furthermore, additional genes can be introduced into the germline using specific promoter/enhancer sequences to generate classical transgenic mice. Several copies of these gene fragments are randomly inserted into the genome and ectopically expressed.

Schäfer et al. [6] have put together a comprehensive review of recent findings using classical knockouts and transgenic mice to investigate acute experimental pancreatitis. Interestingly, there are some mutant mice which show an impaired activation of trypsinogen compared to wild-type controls but histologically a more severe pancreatitis [7]. In another set of experiments, the use of knockouts led to conclusions which differ from the approach using pharmacological inhibitors or antibodies $[8$, 9]. It is obvious that pharmacological substances have led to false conclusions in the past, since these compounds cannot be absolutely specific. The use of genetically defined knockout mice have the advantage of representing a 'clean' approach; however, classical knockout mice have their limitations as well. The presence of a selection marker gene in the targeted locus might affect the mutant phenotype in an unpredictable way. In addition, an inactivated gene may exert its function at several stages of development and in different cell types. This may result in a complex phenotype, where it is difficult to distinguish cell-autonomous from more complex lesions. Furthermore, it has been recently recognized that compensatory mechanisms work in conventional knockouts [10].

Several cellular compartments within the acinar cell are involved in the pathophysiology of acute pancreatitis. Among these cellular structures are zymogen granules and lysosomes. Acinar cells express genes which participate in the defense program and cytokines which may play a role in the initial phase of acute pancreatitis. The burden of circulating cytokines is produced by mononuclear cells and/or macrophages resident in the liver and the lung [1]. Beside acinar cells and macrophages other cell types like endothelial cells and parenchymal cells of other organs have been suggested to play a role in the course of acute pancreatitis. And even more complex, recent evidence suggests that intracellular signals such as $\mathrm{NF}-\kappa \mathrm{B} / \mathrm{Rel}$ activation have a beneficial effect in one cell type and a harmful effect in a different cell type during the same disease process [11].
If we favor acute experimental pancreatitis in the mouse as a suitable model relevant for the human disease, it will be important to develop conditional gene targeting which means gene inactivation strategies in a tissue- and/ or time-specific fashion. To inactivate genes in a tissueand/or time-specific fashion, the bacteriophage-derived Cre-loxP recombination system has been shown to perform well in mouse cell in vivo and appears to offer a broad range of applications $[12,13]$. The Cre enzyme recognizes a sequence motif of $34 \mathrm{bp}$, called loxP. If a DNA segment is flanked by two loxP sites in the same orientation, Cre excises that segment from the DNA, leaving a single loxP site behind. By positioning two loxP sites in introns flanking a critical sequence of the gene of interest, one can use this system to produce a deleted allele. The expression of Cre determines the time point and the tissue specificity of the deletion. When Cre is not expressed, the introduced two loxP sequences in introns should not alter the genes function, its function should be comparable to the wild-type allele. In addition to the generation of conditional knockouts, Cre-loxP recombination system can be used for general mutagenesis including gene replacements, insertions and point mutations [14]. Up to now a large number of mice which harbor genes tagged with two loxP sites, called floxed mice, has been generated and made available to the scientific community. The extent to which conditional gene targeting can be applied to pancreatic research depends on the availability of $\mathrm{Cre}$ mice in which Cre is expressed at sufficient high levels, but under strict cell-type-specific control relevant to the pancreas. Cell-type specificity is conferred by specific promoters. Inducibility is achieved by fusion proteins in which Cre is fused to the ligand-binding domain of a mutated steroid receptor which can be activated by synthetic ligands [15]. At present, a limited number of Cre-mice targeting cells important for the exocrine pancreatic development and function are available. Among these mice are Pdx1-Cre, Ptf1-Cre, Elastase-Cre, and CK19-Cre [1619]. Pdx-1-Cre and Ptf1-Cre delete early in pancreatic development in exocrine and endocrine cells. ElastaseCre in mostly expressed acinar cell and $C K-19$ in expressed in various tissue including duct cells of the pancreas.

This new technology will allow us to evaluate the function of genes and their products in a 'clean' way. Using acinar cell-specific Cre-mice one can determine and functionally test acinar cell-specific defense mechanisms. These approaches will allow us to dissect the role of signaling pathways in a cell type-specific fashion. We will be be able to identify pathways which determine the course 
of the disease. Moreover, it will be possible to identify the role of a signaling molecule in a certain cell type at a certain time point after initiating acute pancreatitis. It will be feasible to determine the contribution of different cell types to systemic cytokine levels and therefore determine key factors for the development of SIRS and MOF. Un- doubtedly, these studies will yield a deeper understanding of the pathophysiology of experimental pancreatitis in mice. Whether this knowledge can be translated to the prevention and clinical management of acute pancreatitis is the ultimate question.

\section{References}

1 Schmid RM, Adler G: Cytokines in acute pancreatitis: New pathophysiological concepts evolve. Eur J Gastroenterol Hepatol 1999;11: 125-127.

2 Duan RD, Zheng CF, Guan KL, Williams JA: Activation of MAP kinase (MEK) and Ras by cholecystokinin in rat pancreatic acini. Am J Physiol 1995;268:G1060-G1065.

3 Dabrowski A, Grady T, Logsdon CD, Williams JA: Jun kinases are rapidly activated by cholecystokinin in rat pancreas both in vitro and in vivo. J Biol Chem 1996;271:5686-5690.

4 Steinle AU, Weidenbach H, Wagner M, Adler G, Schmid RM: Nuclear factor kappa B activation in cerulein pancreatitis. Gastroenterology 1998; 116:420-430.

5 Tando Y, Algül H, Schneider G, Weber CK, Weidenbach H, Adler G, Schmid RM: Induction of IkappaB-kinase by cholecystokinin is mediated by trypsinogen activation in rat pancreatic lobules. Digestion 2002;66:237-245.

6 Schäfer C, Tietz AB, Göke B: Pathophysiology of acute experimental pancreatitis: Lessons from genetically engineered animal models and new molecular approaches. Digestion 2005;71:162-172.

7 Imamura T, Asada M, Vogt SK, Rudnick DA, Lowe ME, Muglia LJ: Protection from pancreatitis by the zymogen granule membrane protein integral membrane-associated protein-1. J Biol Chem 2002;277:50725-50733.
8 Suzuki S, Miyasaka K, Jimi A, Funakoshi A: Induction of acute pancreatitis by cerulein in human IL-6 gene transgenic mice. Pancreas 2000;21:86-92.

9 Cuzzocrea S, Mazzon E, Dugo L, Centorrino T, Ciccolo A, McDonald MC, de Sarro A, Caputi AP, Thiemermann C: Absence of endogenous interleukin-6 enhances the inflammatory response during acute pancreatitis induced by cerulein in mice. Cytokine 2002;18:274285.

10 Sage J, Miller PA, Perez-Mancera, Wysocki JM, Jacks T: Acute mutation of retinoblastoma gene function is sufficient for cell cycle re-entry. Nature 2003;424:223-228.

11 Chen LW, Egan L, Li ZW, Greten FR, Kagnoff MF, Karin M: The two faces of IKK and NFkappaB inhibition: prevention of systemic inflammation but increased local injury following intestinal ischemia-reperfusion. Nat Med 2003;9:575-581.

12 Orban PC, Chui D, Marth JD: Tissue- and sitespecific DNA recombination in transgenic mice. Proc Natl Acad Sci USA 1992;89:68616865.
13 Lakso M, Saur B, Mosinger B, Lee EJ, Manning RW, Yu SH, Mulder KL, Westphal H: Targeted oncogene activation by site-specific recombination in transgenic mice. Proc Natl Acad Sci USA 1992;89:6232-6236.

14 Rajewsky K, Gu H, Kühn R, Betz UAK, Müller W, Roes J, Schwenk F: Perspectives series: molecular medicine in genetically engineered animals: Conditional gene targeting. J Clin Invest 1996;98:600-6003

$15 \mathrm{Gu}$ G, Dubauskaite J, Melton DA: Direct evidence for the pancreatic lineage: NGN3+ cells are islet progenitors and are distinct from duct progenitors. Development 2002;129:24472457.

16 Gannon M, Herrera PL, Wright CV: Mosaic Cre-mediated recombination in pancreas using the $\mathrm{pdx}-1$ enhancer/promoter. Genesis 2000;26:143-144

17 Kawaguchi Y, Cooper B, Gannon M, Ray M, MacDonald RJ, Wright CV: The role of the transcriptional regulator Ptfla in converting intestinal to pancreatic progenitors. Nat Genet 2002;32:128-134.

18 Grippo PJ, Nowlin PS, Cassaday RD, Sandgren EP: Cell-specific transgene expression from a widely transcribed promoter using Cre/ lox in mice. Genesis 2002;32:277-286.

19 Herrera PL, Nepote V, Delacour A: Pancreatic cell lineage analyses in mice. Endocrine 2002; 19:267-278. 